This is the accepted manuscript of:

Capodieci, Nicola, Roberto Cavicchioli, and Andrea Marongiu. "A Taxonomy of Modern GPGPU Programming Methods: On the Benefits of a Unified Specification." IEEE Transactions on Computer-Aided Design of Integrated Circuits and Systems (2021).

(C) IEEE 2021. Personal use of this material is permitted. Permission from IEEE must be obtained for all other uses, in any current or future media, including reprinting/republishing this material for advertising or promotional purposes, creating new collective works, for resale or redistribution to servers or lists, or reuse of any copyrighted component of this work in other works.

The definitive Version of Record was published in https://doi.org/10.1109/TCAD.2021.3082863 


\title{
A Taxonomy of Modern GPGPU Programming Methods: On the Benefits of a Unified Specification
}

\author{
Nicola Capodieci, Member, IEEE, Roberto Cavicchioli, and Andrea Marongiu, Member, IEEE
}

\begin{abstract}
Several Application Programming Interfaces (APIs) and frameworks have been proposed to simplify the development of General-Purpose GPU (GPGPU) applications. GPGPU application development typically involves specific customization for the target operating systems and hardware devices. The effort to port applications from one API to the other (or to develop multi-target applications) is complicated by the availability of a plethora of specifications, which in essence offers very similar underlying functionality. In this work we provide an in-depth study of six state-of-the-art GPGPU APIs. From these we derive a taxonomy of the common semantics and propose a unified specification. We describe a methodology to translate this unified specification into different target APIs. This simplifies crossplatform application development and provides a clean framework for benchmarking. Our proposed unified specification is called GUST (GPGPU Unified Specification and Translation) and it captures common functionality found in compute-only APIs (e.g., CUDA and OpenCL), in the compute pipeline of traditional graphic-oriented APIs (e.g., OpenGL and Direct3D11) and in last-generation bare-metal APIs (e.g., Vulkan and Direct3D12). The proposed translation methodology solves differences between specific APIs in a transparent manner, without hiding available tuning knobs for compute kernel optimizations and fostering best programming practices in a simple manner.
\end{abstract}

Index Terms-GPGPU, Parallel Programming Tools

\section{INTRODUCTION}

Architectural heterogeneity is becoming the reference hardware design paradigm in all computing domains, as it effectively addresses the energy and thermal walls implied by CMOS technology scaling. General Purpose Graphics Processing Units (GPGPU) represent probably the most widespread example of such design paradigm, and GPGPU programming is nowadays a very common paradigm to efficiently execute large data-parallel workloads. The massively parallel compute hardware of modern GPUs is no longer designed for graphicsrelated tasks only, as it was originally. By offloading the execution of compute-intensive, data-parallel code kernels from general-purpose Central Processing Units (CPU) on top of such hardware resources it is possible to hit unprecedented performance-per-watt targets. GPGPU computing has been successfully applied to a variety of performance-demanding computing tasks such as large scale simulations [1, 2], VLSI placement and gate sizing [3, 4], signal processing [5], Machine Learning (ML) [6] and everything else that requires high throughput performance over consistently large sets of input data [7]. Moreover, we are witnessing a trend towards the integration of increasingly powerful GPGPU compute

Department of Physics, Informatics and Mathematics, University of Modena and Reggio Emilia, Italy, e-mail: name.surname@unimore.it. capabilities also at the System-on-Chip (SoC) scale, which enables the execution of machine learning (ML) and Artificial Intelligence (AI) workloads also on top of high-end embedded systems [8].

In order to reach a large variety of users, during the course of the years both the research community and the industry have proposed many high-level languages for simplifying access to such technologies: by providing explicit hooks to the compute pipeline of traditional graphics-oriented APIs (e.g. Khronos OpenGL and Microsoft Direct Compute over Direct3D ), to the release of compute-specific GPU-Accelerated programming models such as NVIDIA CUDA and Khronos OpenCL. All these APIs aim at hiding the complexity of dealing with details specific to the hardware or OS-level drivers. However, while their library-based interface indeed exposes hardware functionality with some degree of abstraction, it is widely agreed that this style of programming is still very involved and, overall, low-level. This has motivated over the last decade a lot of research efforts aimed at further raising the level of abstraction of GPU programming methods.

An application developer chooses a target API based on a number of factors: (i) dependencies from legacy code in a project; (ii) time-to-market constraints and the expertise of the developers; (iii) the need to develop a product meant for multiplatform execution or to port an existing product from one platform to another. Once a program is written with a given API, the effort for porting it on a different API and/or to a different platform is typically not at all trivial. Thus, over the years the research problem of simplifying GPU programming has also tackled the specific issue that porting applications from one GPU system to another, or developing multi-target applications, is complicated by the availability of a large number of low-level APIs. Moving from the observation that these APIs ultimately offer very similar underlying semantics, a number of research and industrial efforts have addressed the need to derive some sort of unified specification/language capable of working across multiple APIs. These approaches and associated tools have proven effective at easing application porting to various GPU HW/SW platforms, but they have so far been very limited and only targeted a few specific APIs.

In this paper we provide an in-depth study of the anatomy of six state-of-the-art APIs for GPGPU programming: OpenGL Compute Shaders, Direct Compute over Direct3D11, OpenCL, CUDA and next-generation bare-metal APIs Vulkan and Direct3D12. Out of this study we derive a taxonomy of the semantics supported across the considered APIs, highlighting how the low-level services underlying virtually every available API for GPU development largely overlap in functionality. 
From there, we discuss and explore the feasibility and the benefits of a unified specification that (i) captures common semantics to any GPGPU-related API consisting of host processor (CPU) and device (GPU) commands; (ii) defines a simple and comprehensive syntax for their deployment. To carry out an early quantitative assessment of the feasibility of such approach, we discuss a proof-of-concept implementation of a unified language front-end, and a methodology to transparently instantiate any of the targeted APIs as language back-ends. The proposed process is literally as simple as recompiling a program for a different target, as the methodology infers the required information from the unified functions and handles internally the differences among APIs. The code produced in this way can then be translated into an executable program using the (unmodified) native compilation flow for any target API. Finally, we provide an evaluation of the core functionality of the provided proof-of-concept implementation, discussing benefits, limitations and how to address them.

\section{RELATED WORK}

Graphic Processing Units were born as hardware components designed with the specific purpose to generate images for visual display. However, since the beginning of the $21 \mathrm{st}$ century programmable GPU pipelines - initially developed for better graphics processing - were found to fit scientific computing needs well, just like the matrix/array data used to formulate such problems. Since then, efforts to use GPUs as general-purpose processors have led to a radical transformation of both their hardware (fully programmable compute units, support for floating-point operations) and their programming methods, which evolved from cumbersome, complete reformulations of computational problems in terms of graphics primitives in the infancy of GPGPU computing, (OpenGL, DirectX) to the advent of truly general-purpose programming languages and APIs (OpenCL, CUDA).

Today, efficiently implementing applications in which a CPU host offloads data to a GPU capable of massively parallel computations is not a trivial task. Programming paradigms such as CUDA or OpenCL have a very involved coding style, which require "being fluent" in low-level device-side languages and related compilation procedures, for interacting with the host. Over the course of the years a plethora of approaches have been proposed to raise the level of abstraction in GPGPU programming, which typically build on top of lowlevel programming models and APIs.

Efforts towards simplifying heterogeneous systems programming can be roughly grouped in two macro-categories, namely (1) libraries of building blocks and (2) language extensions.

A building block is typically referred to as an easy-touse abstraction of mathematical operations commonly adopted across many application domains in parallel calculus. Such operations are usually performed on large input data sets, typically organized in non-trivial data structures such as sparse matrices or graphs. Ease of programming is achieved by exposing an interface in which such function calls are able to hide one or more of the following aspects [9]: writing and compiling compute kernels, organizing the relevant data structures in a GPU friendly manner (i.e., data strides and access pattern should trigger coalesced GPU memory accesses) and memory management in heterogeneous address spaces. Approaches that build on top of such libraries to further raise the level of abstraction have also been explored [10], including SkePU [11], Raja [12], HPX [13] and Kokkos [14].

Language extensions is another well-explored approach to raising the level of abstraction in GPGPU programming. The solutions available in research papers or in real-life development tools pertain to two categories: (i) true language extensions, where standard programming languages and associated compilers have been augmented with new datatypes and keywords to specify parallel execution on a GPGPU; (ii) compiler directives, where the information about where and how to modify the program for parallelization is more pragmatically provided via code annotations, without interfering with the original type system and semantics of the used language. In this category OpenMP is probably the most significant and well-known example. The OpenMP specifications have evolved in the latest releases to simplify the orchestration of computation between host and GPU-like accelerators. OpenACC (Open ACCelerator) ${ }^{1}$ adopts the same philosophy and coding style, and was explicitly designed to simplify the porting of High Performance Compute applications to a wide-variety of heterogeneous hardware. SYCL2 from the Khronos group, is another notable attempt at raising the level of abstraction for heterogeneous systems programming. Attempts to quantify the benefits of the more abstract coding style of such approaches have been made [15], reporting on average about $6.7 \mathrm{x}$ less programming effort when using OpenACC compared to OpenCL, or 3.6x less programming effort when using OpenMP compared to OpenCL, and about 3.1x less programming effort when using OpenMP compared to CUDA. However, the simplified coding style typically comes at the cost of some performance loss, which is very much implementation-dependent [15].

Besides the need for raising the level of abstraction for GPU programming, the research community has also addressed the problem that porting applications from one GPU system to another, or developing multi-target applications, is complicated by the availability of a large number of APIs. Indeed, besides CUDA and OpenCL several other APIs have evolved to become widely adopted standards for graphics rendering (OpenGL, OpenGLES, Direct3D) and general-purpose computing (Vulkan, Direct3D12). Despite its cross-platform nature, OpenCL is not widely supported: a recent analysis targeting Android devices currently available in the market shows that OpenCL support is only available in $32 \%$ of these products [16]. This is, incidentally, the reason why a GPU backend for TensorFlow Lite has been only available as an OpenGLES implementation until very recently ${ }^{3}$. A number of research and industrial efforts have addressed the need to derive some sort of unified specification/language, capable to work across

\footnotetext{
${ }^{1}$ https://www.openacc.org/

${ }^{2}$ https://www.khronos.org/sycl/

${ }^{3}$ An OpenCL implementation has been announced from TensorFlow Lite developers: https://www.tensorflow.org/lite/performance/gpu_advanced
} 
multiple GPU APIs. RenderScript [17], an Android-specific graphics and compute API, features implementations targeting OpenCL, OpenGL and standard CPU as a backend [18].

Intel's newly released oneAPI specification ${ }^{4}$ offers an unified programming model aimed at delivering a common developer experience across accelerator architectures.

Other authors have proposed the idea of unified semantics among different GPGPU APIs targeting OpenCL and CUDA as compilation backends [19], and proposing a framework offering a unified specification with easy-to-use abstractions for managing compute and data resources. Narrowing the application target to tensor operations, other approaches have investigated an abstraction layer for deep neural networks, capable of generating CUDA, OpenCL and Vulkan code [20].

Other approaches have proposed simple languages for specifying device code and a compiler generating highly-optimized CUDA and OpenCL kernels [21] [22]. Similarly, OpenMP and OpenACC implementations for GPGPUs exist [23], targeting CUDA and OpenCL as backends [24]. C++ AMP also features GPU implementations that rely on CUDA, DirectCompute or OpenCL [25] for low-level execution.

All these approaches and tools have proven effective at easing the task of porting applications to various GPU hardware/software platforms; however, they have so far only targeted a few specific APIs. In this paper, we aim at assessing the benefits of extending this type of methodology to all of the most widespread GPU APIs, analyzed in the following.

\section{A TAXonomy of Modern GPU API SEmantics}

In the remainder of this paper we delve into studying the anatomy of six selected low-level APIs. Our aim is to understand which standard features are common to all these APIs, with an interest in exploring whether some sort of unified specification and semantics could be derived, and the benefits this could bring to GPGPU developers.

\section{A. API selection}

Figure 1 shows the main language abstraction of six widespread APIs for handling graphics and compute pipelines of modern GPGPUs. The figure highlights the fact that all the APIs provide features to express general-purpose computation for execution on the GPU (compute pipeline). The focus of our study is on these latter features.

a) CUDA: - CUDA (Compute Unified Device Architecture) is a widely adopted API and programming model for GPGPU computing. It is a NVIDIA proprietary standard firstly released in 2007. Like all the other APIs for programmable GPUs, writing a CUDA application implies describing the interaction between host (CPU) and one or more device accelerators (GPUs). On the host side, the programmer can elect to use the CUDA Driver API or the CUDA Runtime API, which are mutually exclusive in their usage. The CUDA Runtime API eases host code development by providing implicit context initialization and a simplified syntax for launching compute kernels compared to the CUDA Driver API. The CUDA Driver

${ }^{4}$ https://www.oneapi.com/
API offers a higher degree of control over these aspects, at the cost of a more involved coding style. On the device side, parallel computations are described as a grid of threads grouped in blocks, adopting a C++ like syntax enriched with specific keywords used for thread indexing, dynamic kernel invocations, synchronizations etc.

b) OpenCL: - OpenCL (Open Computing Language) ${ }^{5}$ is an industry standard for heterogeneous computing for massively parallel architectures created by Apple in 2009 but nowadays maintained by the Khronos Group. OpenCL presents two major differences compared to CUDA. First, its open nature allows to target generic devices other than NVIDIA GPUs. Second, unlike CUDA, OpenCL provides a more generic API that creates an abstraction layer suitable for a variety of compute accelerators (e.g: FPGA, DSPs, multicore CPUs besides GPUs). This makes developing an OpenCL application slightly harder compared to a CUDA equivalent. Just like CUDA, an OpenCL program is divided between host and device code. On the device-side, the OpenCL specifications allow the developer to describe parallel computations in a high level language (the OpenCL Kernel language) derived from $\mathrm{C} / \mathrm{C}++$ and a standard intermediate binary format (SPIR/SPIR-V 6. Standard Portable Intermediate Representation). Performance-wise, it has been shown that OpenCL and CUDA behave quite similarly [26], with small performance gaps given by the different compilation heuristics employed by the different drivers. Other authors investigated the portability issues of specific kernels from CUDA to OpenCL in [27].

Although OpenCL and CUDA can share buffers to graphic contexts in a seamless manner, they both were specifically designed to be compute-only APIs (i.e., to describe generalpurpose computation).

c) OpenGL: - Among the APIs for real-time graphics rendering, the most representative example is probably OpenGL (Open Graphic Language), a cross-platform standard released in 1992 by Silicon Graphics and currently maintained by Khronos. OpenGL went through a significant evolution over the course of the years. The biggest evolutionary step coincides with the introduction of programmable shaders (to match the hardware evolution from fixed-functionality graphics co-processors to programmable GPUs). Since version 4.3, OpenGL includes the concept of compute shader, namely a pipeline stage targeting the execution of general-purpose compute code, which logically runs alongside rendering stages (e.g., vertex and fragment processing). OpenGL features $d e$ vice vendor extensions, which allow for the development of hardware vendor-specific functionalities not included in the OpenGL standard, and that can be exposed to the application developer. The language for device code development is called GLSL (GL Shading Language). In mid 2003 the Khronos group proposed OpenGLES, a subset of modern OpenGL functionalities specifically designed to run on mobile and embedded systems, hence becoming the most widespread GPU API in history. Starting from version 3.1, OpenGLES fully supports compute shaders.

5 https://www.khronos.org/registry/OpenCL/specs/opencl-2.0.pdf ${ }^{6}$ https://www.khronos.org/registry/spir-v/specs/1.0/SPIRV.pdf 


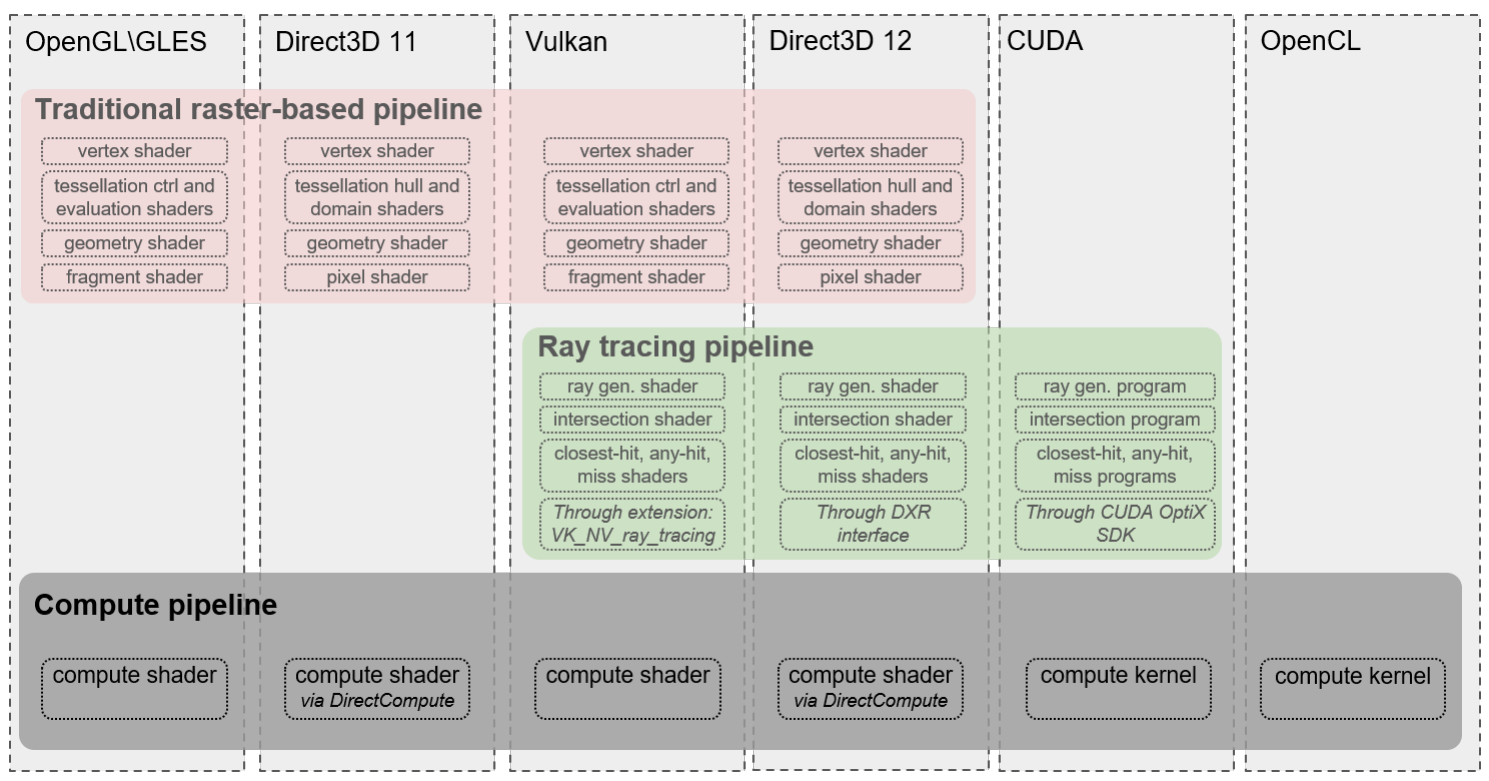

Fig. 1: Summary of GPU APIs programmable functionality set.

d) Direct3D: - Direct3D is a GPU API from Microsoft for graphics rendering on certified Windows platforms. It can be seen as the Windows-only OpenGL counterpart and, compared to the Khronos standard, it exposes a thinner abstraction layer. Moreover, it does not feature the equivalent of OpenGL extensions. The Direct3D equivalent for a GL compute shader exploits the DirectCompute technology. Device code within a Direct3D program is written using HLSL (High Level Shading Language). In this work we examine two major Direct3D releases: Direct3D11 and the recently released Direct3D12. The differences between the Direct3D11 and Direct3D12 are major, to the point that Direct3D 12 cannot be considered the Windows counterpart of OpenGL anymore, but rather that of another recently released Khronos Standard; Vulkan.

e) Vulkan: - Vulkan is a recent open and cross-platform standard for GPU programming. It was proposed and now maintained by the Khronos group. It is described as both a graphics and a compute API as the Vulkan programming model is agnostic to which of these two pipelines will be mostly used in an application. Vulkan is getting widely supported across different hardware and operating systems. In a recent announcement, Google stated that a working Vulkan implementation will be required on all 64-bit devices running Android Q. This suggests that Vulkan has been designed to be the successor of both OpenGL and OpenGLES.

Vulkan and Direct3D12 differ from the rest of the APIs covered here in the way they handle driver interactions. Rather than relying on a constant driver interaction for error checking, hidden optimizations and other operations (for which the application developer has little to no control), Vulkan and Direct3D12 are much more explicit in the way in which commands are submitted to the GPU driver. In such model, all the low-level details of computation offloading (including synchronization, error checking, etc.) are exposed to the programmer, which has to take care of these aspects manually.

\section{B. A note on the adopted terminology}

In the remainder of this paper we refer to CUDA, OpenCL, OpenGL and Direct3D11 as the Traditional APIs, whereas Vulkan and Direct3D12 are referred to as the Command Listbased APIs. Different APIs have different names for the same software artifacts, hence we establish here common terminology to avoid ambiguities.

First, we define the concept of context. A context represents the abstraction of an API-specific resource manager. A context has to be created once at the beginning of the application and released upon completion. A context also represents the interface in which GPU-related software artifacts might be read and modified.

Concerning the code to be executed on the GPU, the terms compute shader, compute kernel or compute program are indistinctly chosen, and sometimes abbreviated in shader, kernel or program. When a shader, program or kernel is launched on the GPU we refer to this operation as invocation or dispatch indistinctly.

A kernel has input and output data in the form of arguments and symbols. To understand the difference, let us consider the following CUDA 7 kernel function signature:

$$
\begin{array}{r}
\text { _global__ void vector_add(const int } A \text {, } \\
\text { const int* } B \text {, int* C, int N); }
\end{array}
$$

The device function vector_add takes four inputs: two pointers $\mathrm{A}$ and $\mathrm{B}$ to the input vectors (also called data buffers), a pointer $\mathrm{C}$ to the output vector, and the integer $\mathrm{N}$, that represents the vectors' size. While OpenCL works in a very similar manner to this CUDA example, other APIs not only adopt different terminology to distinguish between pointers to buffers and constant values, but they also need special operations for binding inputs to one ore more specific programs. In our chosen terminology, A, B and C are called arguments or resources and $\mathrm{N}$ is called a symbol. Arguments

\footnotetext{
${ }^{7}$ The concepts illustrated are not specific to CUDA.
} 
TABLE I: API specific Host-side operations for the considered APIs

\begin{tabular}{|c|c|c|c|c|c|c|}
\hline $\begin{array}{l}\text { Operation - } \\
\text { API }\end{array}$ & CUDA Driver API & OpenCL & $\begin{array}{l}\text { OpenGL } \\
\text { Compute Shaders }\end{array}$ & $\begin{array}{l}\text { DirectCompute } \\
\text { over Direct3D } 11\end{array}$ & Vulkan & $\begin{array}{l}\text { DirectCompute } \\
\text { over Direct3D } 12\end{array}$ \\
\hline $\begin{array}{l}\text { Context } \\
\text { Creation }\end{array}$ & $\begin{array}{l}\text { cuInit } \\
\text { cuDeviceGet } \\
\text { cuCtxCreate }\end{array}$ & $\begin{array}{l}\text { clCreateContext } \\
\text { FromType }\end{array}$ & $\begin{array}{l}\text { Platform specific } \\
\text { sys. calls }\end{array}$ & D3D11CreateDevice & $\begin{array}{l}\text { vkCreateInstance } \\
\text { vkCreateDevice }\end{array}$ & D3D12CreateDevice \\
\hline $\begin{array}{l}\text { Kernel } \\
\text { Compilation }\end{array}$ & $\begin{array}{l}\text { nvrtcCreateProgram } \\
\text { nvrtcCompileProgram } \\
\text { nvrtcGetPTX } \\
\text { cuModuleLoadDataEx } \\
\text { cuModuleGetFunction }\end{array}$ & $\begin{array}{l}\text { clCreateProgram } \\
\text { WithSource } \\
\text { clBuildProgram } \\
\text { clCreateKernel }\end{array}$ & $\begin{array}{l}\text { glCreateShader } \\
\text { glCompileShader } \\
\text { glCreateProgram } \\
\text { glAttachShader } \\
\text { glLinkProgram }\end{array}$ & $\begin{array}{l}\text { D3DCompileFromFile } \\
\text { ID3D11Device:: } \\
\text { CreateComputeShader }\end{array}$ & vkCreateShaderModule & $\begin{array}{l}\text { D3DCompileFromFile } \\
\text { ID3D12Device:: } \\
\text { CreateComputeShader }\end{array}$ \\
\hline $\begin{array}{l}\text { Arguments } \\
\text { allocations }\end{array}$ & $\begin{array}{l}\text { cuMemAllocHost } \\
\text { cuMemAlloc }\end{array}$ & $\begin{array}{l}\text { clCreateBuffer } \\
\text { clEnqueueMapBuffer }\end{array}$ & $\begin{array}{l}\text { glGenBuffer } \\
\text { glBindBuffer } \\
\text { (SSBO) }\end{array}$ & $\begin{array}{l}\text { ID3D11Device:: } \\
\text { createBuffer. } \\
\text { SRV or UAV }\end{array}$ & vkCreateBuffer & $\begin{array}{l}\text { ID3D12Device:: } \\
\text { Create*Resource }\end{array}$ \\
\hline Symbols & $\begin{array}{l}\text { const values in } \\
\text { kernel invocation }\end{array}$ & $\begin{array}{l}\text { const values in } \\
\text { kernel invocation }\end{array}$ & OpenGL uniforms & Constant Buffers & Push Constants & Constant Buffers \\
\hline $\begin{array}{l}\text { Buffer } \\
\text { Synchr. }\end{array}$ & cuMemCpy*Async & clEnqueue**Buffer & $\begin{array}{l}\text { glBufferData } \\
\text { glMap/Unmap } \\
\text { \& memcpy }\end{array}$ & $\begin{array}{l}\text { D3D11_MAPPED_ } \\
\text { SUBRESOURCE } \\
\text { map/unmap } \\
\text { \& memcpy }\end{array}$ & vkCmdCopy* & $\begin{array}{l}\text { ID3D12Graphics } \\
\text { CommandList:: } \\
\text { CopyBufferRegion }\end{array}$ \\
\hline $\begin{array}{l}\text { Arguments } \\
\text { binding }\end{array}$ & $\begin{array}{l}\text { in kernel invocation } \\
\text { cuLaunchKernel }\end{array}$ & clSetKernelArg & glBindBufferBase & $\begin{array}{l}\text { ID3D11DeviceContext1:: } \\
\text { CSSetShaderResources } \\
\text { CSSetUnorderedAccess } \\
\text { Views }\end{array}$ & $\begin{array}{l}\text { Descriptor Sets and } \\
\text { Layouts }\end{array}$ & Root Signature Descr. \\
\hline $\begin{array}{l}\text { Launch } \\
\text { Config. }\end{array}$ & $\begin{array}{l}\text { in kernel } \\
\text { invocation } \\
\text { cuLaunchKernel }\end{array}$ & $\begin{array}{l}\text { in kernel invocation } \\
\text { clEnqueueNDRange } \\
\text { Kernel }\end{array}$ & $\begin{array}{l}\text { in dispatch call } \\
\text { glDispatchCompute } \\
\text { GroupSize (ARB ext.) } \\
\text { glDispatchCompute } \\
\end{array}$ & $\begin{array}{l}\text { in dispatch call } \\
\text { ID3D11DeviceContext1:: } \\
\text { Dispatch }\end{array}$ & $\begin{array}{l}\text { Specialization Consts. } \\
\text { dispatch call } \\
\text { vkCmdDispatch }\end{array}$ & $\begin{array}{l}\text { In dispatch Call } \\
\text { ID3D12Graphics } \\
\text { CommandList:: } \\
\text { Dispatch } \\
\end{array}$ \\
\hline $\begin{array}{l}\text { Device } \\
\text { Wait For Idle } \\
\text { (WFI) }\end{array}$ & cuCtxSynchronize & $\begin{array}{l}\text { clFlush } \\
\text { clFinish }\end{array}$ & $\begin{array}{l}\text { glFlush } \\
\text { glFinish }\end{array}$ & $\begin{array}{l}\text { blocking wait on } \\
\text { a ID3D11Query }\end{array}$ & vkDeviceWaitIdle & $\begin{array}{l}\text { blocking wait on a } \\
\text { ID3D12Fence }\end{array}$ \\
\hline
\end{tabular}

can be read-only, write-only or might allow both operations. Symbols represent read-only data to the device (only written by the host). Every API has to bind sets of arguments and symbols to one or more kernels: we refer to this operation as setting the kernel layout.

Also related to kernels, a program invocation needs a launch configuration, which logically describes the degree of parallelism in which the work must be computed over a grid of parallel threads. According to the dimension of the compute program data set, such grid might be designed to expand along 1,2 or 3 dimensions. Every API therefore exposes a way to dispatch a kernel using a specific configuration of threads and groups of threads over each dimension. To this respect, we adopt the CUDA terminology: a GPU computation is divided into threads and threads are grouped into blocks or groups.

Buffers hosting data passed via function arguments might be allocated both on the host side and on the device side. When a host pointer has a corresponding device pointer, communication might be more or less explicitly synchronized. This implies a device-to-host or a host-to-device copy. Under the Unified Memory Model, a paradigm aimed at simplifying heterogeneous programming by hiding the existence of distinct address spaces (CPU and GPU), a buffer pointer can be flagged to allow unified access from both the host and device, without requiring explicit copies/synchronization [28]. We further discuss unified memory in Section III-C3

Specific to the Command List-Based APIs we define the terms Pipeline State Object (PSO) and command buffer. A PSO is a pre-compiled description of settings for specific kernels, and this includes arguments and symbols bindings as well as launch configurations. A command buffer is a pre-recorded set of commands (PSO selection, kernel invocations and data buffer movements). Command List-Based APIs require that low-level offload operations are explicitly handled in advance to minimize driver interactions during the runtime execution of the applications (see Section III-C2).

\section{API Constructs for Host Code}

In this section we isolate and study the "lowest common denominator" for host-side operations performed in all the GPU APIs, from context initialization to kernel dispatch.

1) Traditional APIs: Besides context creation, in traditional APIs the developer has to take care the following aspects: kernel compilation, allocation of buffers for arguments, definition of symbols, buffer synchronization (i.e., data transfers), arguments binding (kernel layout) and the actual dispatch computations with their related launch configurations. The first four columns of Table I summarize how all the considered traditional APIs perform these operations.

Table Imaps each host-side operation (from context creation to dispatch call) and other relevant software constructs to respective API-specific terminology and function calls. For CUDA, we consider the Driver API rather than the Runtime $A P I$, as - functionally speaking - the latter is merely a C++ wrapper around the C-based Driver API. For all these APIs, a kernel source code can be extracted from a file or might be hosted in a string variable.

Context creation exploits API-specific function calls, OpenGL being the only exception. The OpenGL specification states that context creation is not regulated by the Khronos standard: this implies calling platform-specific system calls as opposed to regular $g l \star$ function calls. Allocation functions regulate memory allocations for both host and device data, according to configurable access flags. A device-side buffer is stored as an API-specific data structure, like CUDeviceptr for CUDA or Cl_mem for OpenCL. In OpenGL, generic device-visible data are Shader Storage Buffer Objects $\$$ (SSBOs). In Direct Compute on Direct3D11, Shader Resource Views (SRVs) are used for read-only device side buffers and Unordered Access Views (UAVs) for those buffers that need to be read back by the host.

\footnotetext{
${ }^{8}$ https://www.khronos.org/opengl/wiki/Shader_Storage_Buffer_Object
} 
Symbols are trivially implemented for both CUDA and OpenCL; the OpenGL equivalent are called uniforms, whereas on Direct Compute-based APIs the functional equivalent are Constant Buffers (CBs).

Other functionality shown in Table II is straightforward to address, with the exception of the launch configuration part, which requires additional remarks. While none of the APIs has any limitation in dynamically specifying the number of blocks for each grid dimension, problems arise if the user also wants to dynamically specify the number of threads within a block. For CUDA and OpenCL such functionality is trivially implemented; for OpenGL, this is achieved by invoking a compute shader with glDispatchComputeGroupSizeARB instead of glDispatchCompute function. For Direct Compute over Direct3D11, dynamically specifying variable block sizes is not supported: a possible workaround for this is to create the compute shader at runtime, specifying the block size with the HLSL numthreads builtin macro and deferring the shader compilation until just before its first dispatch call.

2) Command List-Based APIs: Command list-based APIs in addition allow (i) to mark specific code sections for constructing PSOs and command buffers and; (ii) the actual submission of the command buffer(s) to the GPU. The last two columns of Table $[$ show the operations and primitives required from context creation to command list submission for the Direct3D 12 and the Vulkan API. Focusing on Direct3D-based APIs, the biggest difference in version 12, compared to version 11 is the necessity of pre-compiling a PSO and populating a command list before the actual dispatch call. Other then that, device code is compiled and described in the same manner. Copy and compute commands are therefore deferred, as all the function calls from the ID3D12GraphicsCommandList interface are only allowed during a command buffer recording stage. The Vulkan equivalents of copy and dispatch operations use the $v k C m d \star$ prefix. These functions can also only be invoked during the recording of a command buffer (VkCommandBuffer data type). This significantly adds to the complexity of writing a general-purpose program.

Vulkan device code is written using a low-level intermediate representation (SPIR-V), which can be compiled via the vkCreateShaderModule function. Third-party toolchains can be used to allow the use of higher level languages (such as HLSL or GLSL) augmented with specific Vulkan extensions. In the following, we assume that an additional compilation step is put in place to allow the description of device-side computations in a Vulkan using GLSL. This allows to treat Vulkan and OpenGL device code as conceptually equivalent for our purposes. We will further elaborate on this assumption.

3) Unified memory: Unified memory models are present in all the examined APIs. CUDA UVM (Unified Virtual Memory) describes a model in which specific host-visible pointers are managed by the CUDA driver through an on-demand page migration mechanism between the CPU and the GPU address spaces [29]. Recent CUDA versions allow the developer to suggest to the GPU driver the actual residency aspects of each allocations (cudaMemAdvise) and even to control memory pages prefetching (cudaMemPrefetch $*$ ).

In recent OpenCL versions, different implementations of the SVM (Shared Virtual Memory) abstraction allow the developer to exploit different granularity for resources shared by the CPU and the GPU. More specifically, starting from OpenCL version 2.0 three types of SVM allocations are available: (i) coarse-grained buffer SVM: entire buffers that reside in devicelocal memory might be accessed by the CPU; (ii) fine-grained buffer SVM: individual loads and stores within OpenCL buffers residing in device-local memory are shared between CPU and GPU; (iii) fine-grained system SVM: sharing between CPU and GPU occurs at the granularity of individual loads/stores within host memory allocations.

Both CUDA and OpenCL in their most recent versions allow the developer to allocate buffers in CPU and GPU space explicitly (cudaMallocManaged in CUDA and ClSVMAlloc in OpenCL) or implicitly, i.e. using regular host-side allocation functions (e.g. new and malloc). CUDA and OpenCL offer very fine grained control over page migrations, CPU-GPU cache coherency and over-subscription of video memory: OpenGL and Direct3D11 present a limited control over such aspects.

OpenGL and Direct3D 11 rely on glMapBuffer $\star$ and ID3D11DeviceContext: :Map, respectively, to enforce CPU-only access to a given buffer. The dual unmapping operation allows the sole GPU to (coherently) access that same buffer. Unified memory models delegate to the GPU driver the details of the CPU-GPU coherency among shared buffers. This allows for supporting unified memory also in absence of dedicated hardware. In this case, the GPU driver provides a safe fallback strategy, where different address spaces are transparently managed via implicit buffer copies.

Command list-based APIs introduce the concept of explicit memory management. The programmer is responsible for selecting the appropriate heap for allocating CPU or GPU buffers, thus controlling specific memory types and alignment rules. Different heaps are usually identified by numbers, and have specific residency and coherency rules. Although naming and numbering might slightly change between Vulkan and Direct3D12, without loss of generality we can identify three heaps: (i) Heap 0 - Standard device-only access: allocations that require explicit synchronization and memory copying; (ii) Heap 1 - Device memory accessible by the CPU: allocations that reside on GPU local memory, but that can be made visible to and coherently shared with the CPU; (iii) Heap $2-$ $C P U$ memory accessible by the GPU: allocations that reside on host memory and that are occasionally accessed by the GPU. According to the selected memory type, different cache coherency mechanisms are in place.

For heaps 0 and 1, Direct3D12 and Vulkan allow oversubscription, but the success of these operations ultimately depends on the actual GPU driver implementation. The GPU hardware and driver might not support all the combinations of heaps and memory types: attempting to allocate a specific type on a specific heap within a system that does not support such a combination will fail. API-specific unified memory features are further detailed in Table II 
TABLE II: Unified Memory model implementations for the considered APIs

\begin{tabular}{|c|l|l|}
\hline \multirow{2}{*}{ API } & \multicolumn{1}{|c|}{$\begin{array}{c}\text { Unified Memory } \\
\text { model }\end{array}$} & \multicolumn{1}{c|}{$\begin{array}{c}\text { Description / } \\
\text { supported features }\end{array}$} \\
\hline \multirow{2}{*}{ CUDA } & Kepler and Maxwell GPU uArch & Very basic implementation. Moves dirty pages at kernel launch \\
\cline { 2 - 3 } & post Maxwell & $\begin{array}{l}\text { Prefetching, residency hints, concurrent access, oversubscription } \\
\text { on-demand page migration and system-wide atomics }\end{array}$ \\
\hline \multirow{2}{*}{ OpenCL } & Coarse-Grained buffer SVM & Entire buffers that resides in device memory are shared. Oversubscription \\
\cline { 2 - 3 } & Fine-Grained buffer SVM & Individual loads and stores in device memory are shared. Oversubscription \\
\cline { 2 - 3 } & Fine-Grained system SVM & Individual loads and stores in buffers residing in host memory are shared \\
\hline $\begin{array}{l}\text { OpenGL and } \\
\text { Direct3D 11 }\end{array}$ & $\begin{array}{l}\text { API-Specific Mapping functions } \\
\text { controlled by access flags }\end{array}$ & Allows oversubscription and limited control over residency and coherency \\
\hline \multirow{2}{*}{$\begin{array}{l}\text { Vulkan and } \\
\text { Direct3D 12 }\end{array}$} & Heap 1: Device memory & $\begin{array}{l}\text { Memory type 1: Oversubscription, CPU writes are write-combined and write directly into GPU memory, } \\
\text { whereas CPU reads are uncached. }\end{array}$ \\
\cline { 2 - 3 } & Heap 2: Host memory & $\begin{array}{l}\text { Memory type 2: CPU writes are write-combined, CPU reads are uncached. } \\
\text { Memory type 3: CPU reads and writes go through CPU cache hierarchy, whereas } \\
\text { GPU is able to snoop CPU cache }\end{array}$ \\
\hline
\end{tabular}

TABLE III: Device-side constructs for all the considered GPU APIs

\begin{tabular}{|l|l|l|l|l|}
\hline $\begin{array}{l}\text { Keyword - } \\
\text { Functionality }\end{array}$ & CUDA & OpenCL & GLSL (Vulkan and GL) & HLSL (D3D11 \& 12) \\
\hline local Thread indexing & threadIdx.x,y,z & get_local_id(0,1,2) & gl_LocalInvocationID.x,y,z & SV_DispatchThreadID.x \\
\hline Group-Thread indexing & blockIdx.x,y,z & get_group_id(0,1,2) & gl_WorkGroupID.x,y,z & SV_GroupID.x,y,z \\
\hline Group-Thread size & blockDim.x,y,z & get_work_dim(0,1,2) & gl_LocalGroupSizeARB.x,y,z & statically specified \\
\hline Local Memory & _shared_ & - local & shared & groupshared \\
\hline InterThread synchr. & _synchthreads & $\begin{array}{l}\text { barrier } \\
\text { (CLK_LOCAL_MEM_FENCE) } \\
\text { (CLK_GLOBAL_MEM_FENCE) }\end{array}$ & memoryBarrierShared & GroupMemoryBarrier \\
WithGroupSync & atomicAdd & InterlockedAdd \\
\hline Atomic Add & atomicAdd & atomic_add & & \\
\hline
\end{tabular}

\section{API constructs for Device code}

Any GPGPU application needs device code to describe parallel computation. Different APIs provide different approaches to specifying such code and, similarly to what we highlighted for the host code, it is possible to identify the underlying common semantics to various APIs, concerning abstractions such as thread indexing, local memory management and synchronization (memory barriers, fences and other deviceonly synchronization points). Table III shows an overview of the most preeminent language features that control such semantics for the various APIs.

This table is of course far from being complete; however, the listed constructs and keywords constitute the minimum subset required for efficiently implementing all the parallel primitives typically used to build a full-fledged heterogeneous application [30].

\section{On the Benefits of a Unified Specification: A CAse Study}

In this section, we aim at assessing the benefits of a unified specification across all of the most widespread GPU APIs, that we have already analyzed. This has the potential to provide the most flexible support for heterogeneous crossplatform development. To practically conduct this type of assessment, we first propose a unified specification for device and host code programming, and then provide a proof-ofconcept implementation.

We call this unified specification GUST (GPGPU Unified Specification and Translation), which in addition to defining the common API also mandates the translation rules that allow to target each of the considered low-level APIs as a compilation backend.

\section{A. Introducing GUST}

$\boldsymbol{G U S T}$ exposes an interface that defines a function call for each of the basic operations we identified in section III Due to the substantially different development philosophy characterizing the two API categories introduced in section III (Traditional APIs and Command List-Based APIs), the basic interface exposed by $\boldsymbol{G} \boldsymbol{U} \boldsymbol{S T}$ does not define a common layer between the two, as we believe the expert user should not be prevented from explicitly leveraging the low-level constructs and optimization knobs exposed by Command List-Based APIs. We will briefly discuss in Section $\mathrm{V}$ how a unification layer between Traditional APIs and Command List-Based APIs could be achieved practically. Starting from Tables I and III it is in many cases straightforward to remap keywords and function calls across APIs for each entry of the tables. On the host-side, the GUST API derived from the taxonomy in Table I is shown in Table IV The operations described in Table IV can be easily mapped to the previous table entries. Note that the last three columns of table IV refer to the command listbased APIs. More specifically, the functionality extracted from Vulkan and Direct3D 12 allows the developer to specify code sections that mandate where specific $\boldsymbol{G} \boldsymbol{U S T}$ wrapper functions can be called: setting launch configurations, arguments and symbol bindings to specific shaders can only occur within a code block that starts with startCreateP SO and ends with finalizePSO function calls, whereas kernel launches, PSO selection and data transfer operations can only be called in a startCreateCmdList - finalizeCmdList code block. We refer to these code blocks as pipeline creation and command list recording blocks. The pipeline creation code block outputs a PSO handle in which a description of a kernel launch is prepared in advance. The command list recording block stores in advance the actual commands to be later executed by the GPU, as this latter command buffer will 
TABLE IV: Host-side $\boldsymbol{G} \boldsymbol{U} \boldsymbol{S} \boldsymbol{T}$ unified semantics from translation rules

\begin{tabular}{|l|l|l|l|l|l|l|l|l|l|}
\hline Host side operation & $\begin{array}{l}\text { Context } \\
\text { Creation }\end{array}$ & $\begin{array}{l}\text { Compile } \\
\text { Compute Prog. }\end{array}$ & $\begin{array}{l}\text { Resource } \\
\text { Allocation and } \\
\text { synchronization }\end{array}$ & $\begin{array}{l}\text { Argument } \\
\text { binding }\end{array}$ & $\begin{array}{l}\text { Symbol } \\
\text { copy }\end{array}$ & $\begin{array}{l}\text { Launch Conf. } \\
\text { definition }\end{array}$ & $\begin{array}{l}\text { Launch } \\
\text { Program }\end{array}$ \\
\hline GUST API func. call & createCtx & compileProg & $\begin{array}{l}\text { allocate } \\
\text { synchBuffer }\end{array}$ & setArg & $\begin{array}{l}\text { copySymbol } \\
<\mathrm{T}>\end{array}$ & $\begin{array}{l}\text { setLaunch } \\
\text { Configuration }\end{array}$ & $\begin{array}{l}\text { PSO rec. } \\
\text { block } \\
\text { cmd buf. }\end{array}$ \\
rec. block
\end{tabular}

TABLE V: API specific functions and terminology for $\boldsymbol{G} \boldsymbol{U} \boldsymbol{S T}$ unified semantics for device code: preamble definition

\begin{tabular}{|c|c|c|c|c|c|}
\hline Preamble Definition & CUDA & OpenCL & GLSL (GL) & GLSL (Vulkan) & HLSL (D3D11 \& 12) \\
\hline Entry Point & $\begin{array}{l}\text { global__ } \\
\text { void kernelname } \\
(\operatorname{args...)}\end{array}$ & $\begin{array}{l}\text { _kernel void } \\
\text { kernelname } \\
\text { (args...) }\end{array}$ & void main() & void main() & $\begin{array}{l}\text { void kernelname } \\
\text { (launch } \\
\text { configuration) }\end{array}$ \\
\hline $\begin{array}{l}\text { Local memory } \\
\text { static allocation }\end{array}$ & Inside kernel func. & Inside kernel func & $\begin{array}{l}\text { Outside kernel } \\
\text { func. }\end{array}$ & $\begin{array}{l}\text { Outside kernel } \\
\text { func. }\end{array}$ & $\begin{array}{l}\text { Outside kernel } \\
\text { func. }\end{array}$ \\
\hline Symbols and buffers & In kernel args & In kernel args & in layout definition & in layout definition & in layout definition \\
\hline Symbols are defined as & $\begin{array}{l}\text { Single values as } \\
\text { kernel input } \\
\text { arguments }\end{array}$ & $\begin{array}{l}\text { Single values as } \\
\text { kernel input } \\
\text { arguments }\end{array}$ & uniforms & Push constant & $\begin{array}{l}\text { Elements of a } \\
\text { contant buffer }\end{array}$ \\
\hline Buffers are defined as & Device buffers & Device buffers & SSBOs & Storage buffers & Structured Buffers \\
\hline $\begin{array}{l}\text { Symbols/Buffers } \\
\text { Location }\end{array}$ & $\begin{array}{l}\text { Index of an array } \\
\text { of args }\end{array}$ & $\begin{array}{l}\text { Index of an array of } \\
\text { args }\end{array}$ & $\begin{array}{l}\text { Uniform Location } \\
\text { and SSBO bindings }\end{array}$ & $\begin{array}{l}\text { Descriptor sets and } \\
\text { layout bindings }\end{array}$ & $\begin{array}{l}\text { Root signature/ } \\
\text { shader resource slot }\end{array}$ \\
\hline
\end{tabular}

be offloaded with the submitWork member function. All the other functions (resource allocation, kernel compilation, etc.) might be called anywhere outside these blocks. Violating these constraints should return explanatory errors to the user.

Clearly, Table III alone cannot capture all the different mechanics for the observed APIs. Different device-side languages define different entry points, have distinct ways to define data structures and respective links to the host side data. CUDA and OpenCL, for instance, define entry points to device functions that can be called from the host using specific keywords (_olobal_ for CUDA, _ kernel for OpenCL); the kernel layout and related data structures are simply the arguments of such functions. The same aspect is treated differently in all the other APIs: GLSL for instance typically imposes a main function as the entry point for a particular compute shader, forcing the developer to define the layout (data structure description of input and output resources and symbols) outside the shader function. A practical implementation of $\boldsymbol{G U S T}$ 's device-side abstractions must deal with the handling of symbols' and resources' locations. Regarding layout locations, CUDA and OpenCL kernel invocation functions take an array of arguments as input, hence in these cases an argument location is simply their index within the array. In device languages derived from graphic APIs, on the contrary, the concept of input and output argument of a shader is decoupled for the concept of compute program, forcing the programmer to reason about the idea to utilize similar data layouts for different compute programs. Such information constitutes the preamble of compute shaders in GLSL and HLSL, with minor variations among OpenGL/Vulkan and D3D11/D3D12. This is shown in Table V, which summarizes the translation rules for correctly generating a preamble in a cross-platform translation layer for device-side code.

\section{B. A GUST Reference Implementation}

We present a proof-of-concept implementation of $\boldsymbol{G} \boldsymbol{U S T}$. This implementation serves to conduct our case study, and is thus far from being complete. We cover all the key host- and device-side operations previously described. Implementing the $\boldsymbol{G U S T}$ interface for the host-side interactions can be conveniently achieved in the form of a runtime library or the methods of a C++ class. We choose the latter. Listings 1 and 2 show an example 2D histogram computation coded in $\boldsymbol{G} \boldsymbol{U S T}$.

\section{1) Host-Side Operations:}

a) Context Creation: Context creation is straightforwardly implemented for each GPU API as indicated in Table [ There are some additional implementation details to cover for OpenGL and the command list-based APIs. For the OpenGL wrapper, a context is established with the $g l \mathrm{fw}$ library 9 while for core profile library function loading, $g l 3 w$ is used 10 We do this as the OpenGL standard does not regulate the creation of a GL context, leaving this issue to be solved by the individual operating systems. Context creation for both D3D12 and Vulkan requires to specify in advance the memory footprint of the application (in terms of resource usage). This allows to pre-allocate Persistent Staging Buffers (PSBs). A PSB is a buffer whose dimension is set at context creation time and is persistently mapped (until context destruction) within the GPU driver: it represents a staging area in which data to and from the device can be read or written by the application, exploiting the maximum available memory bandwidth without wasting time in mapping and unmapping different buffers when data needs to be moved. When a device-side allocation is requested, a pointer within the range of possible addresses from the PSBs is returned. Compared to traditional APIs, this implies managing a local allocation table with its segmentation logic. GUST can take care of this transparently. For all the APIs, the $\boldsymbol{G U S T}$ initializes the context with the createCtx function call, that initializes with default values any API-specific setting (e.g: PSB size for command list based APIs or preferred device type for OpenCL). Context creation is where $\boldsymbol{G} \boldsymbol{U S T}$ selects the corresponding API wrapper; no other $\boldsymbol{G U S T}$ function calls are permitted before the successful creation of a context.

b) Compute program compilation: The compileProg function call takes care of compiling GUST device code.

\footnotetext{
chttp://www.glfw.org/

1thttps://github.com/skaslev/gl3w
} 
Listing 1: Host-side GUST code for 2D Histogram.

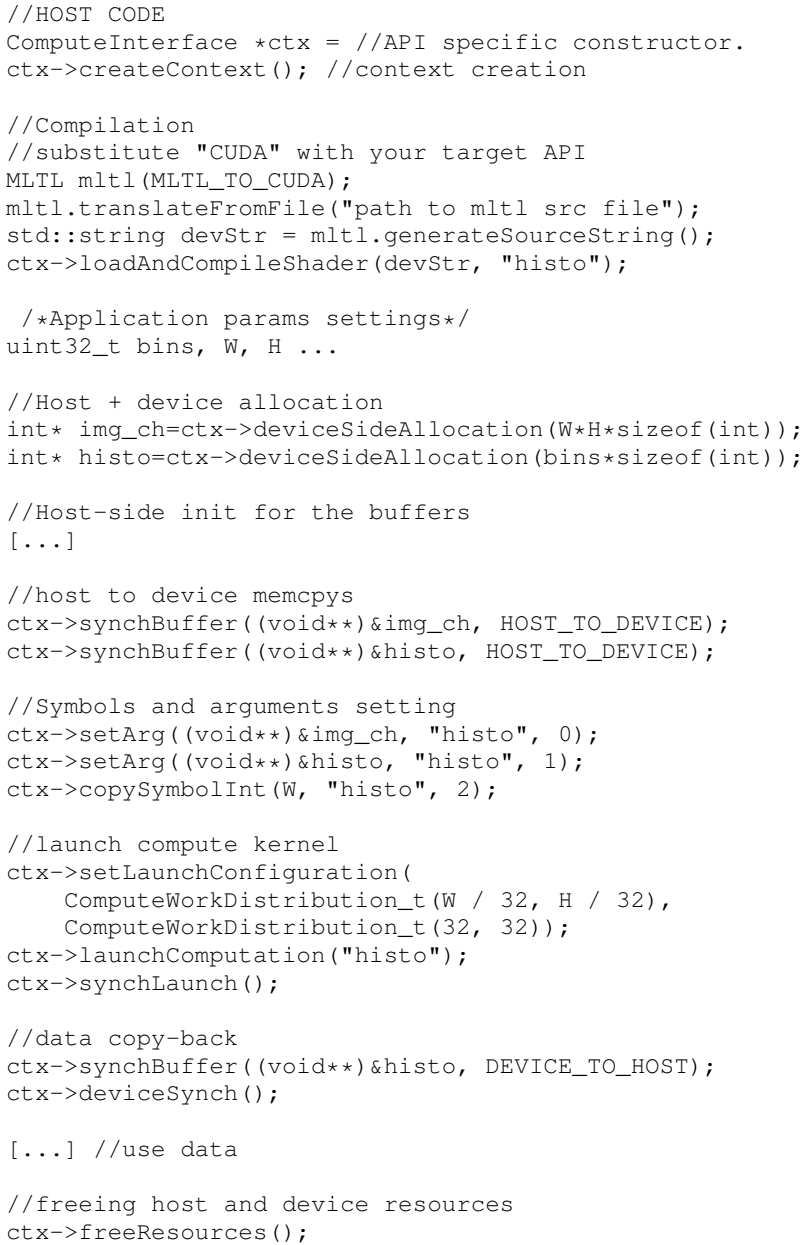

Device code can be stored in a string or in a file and might be specified using a tag-based language implementation of an API-aspecific device code. We call our instance of such a language the MLTL (Metal Layer Translation Language). Internally, GUST translates MLTL code into API-specific code, as API selection already occurred during context creation. Once device-specific code is available, GUST wraps compiling functions as described in table I Instead, using the recently released NVRTC ${ }^{11}$ (NVIDIA Runtime Compilation Library) we are able to create and compile kernels at runtime, hence uniforming the CUDA behavior to all the others APIs. For command list-based APIs, compiling a kernel in D3D12 is done in a very similar manner as seen in D3D11, but for Vulkan an additional step is needed. Using the Valve's LunarG Vulkan SDK ${ }^{12}$ and by exploiting its glslangValidator executable the user is able to feed $\boldsymbol{G} \boldsymbol{U S T}$ with a string or a file containing a GLSL compute shader with Vulkanspecific extension and obtain a SPIR-V source. From that, a vkShaderModule can be created.

c) Resource Management: GUST offers an allocate function to wrap backend API-specific allocation functions.

${ }^{11}$ http://docs.nvidia.com/cuda/nvrtc/index.html

${ }^{12}$ https://www.lunarg.com/vulkan-sdk/
Listing 2: Device-side GUST code (MLTL) for 2D histogram.

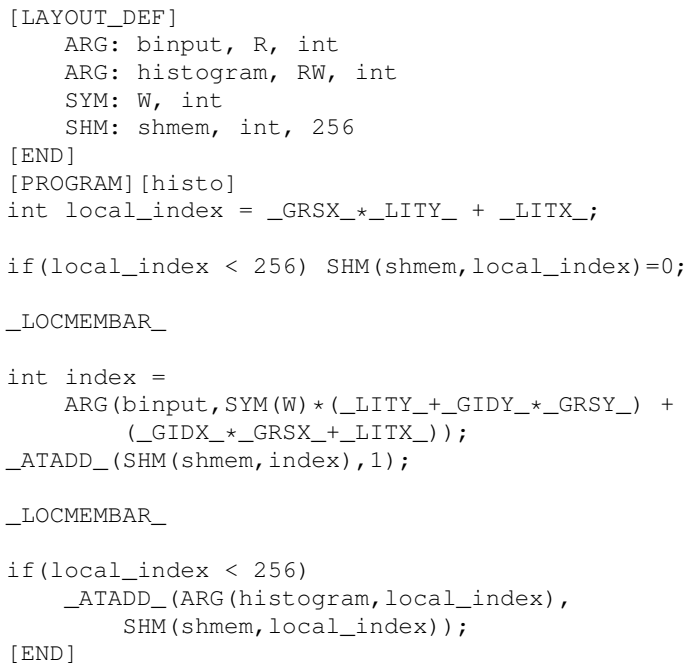

In our example implementation, an allocation call always returns a pointer able to be read and written by the host. Internally, GUST maintains an allocation table in which for each host pointer a corresponding device pointer is present: in this way, the complexity of dealing with two address spaces (GPU and CPU) is partially hidden. However, resources must be explicitly synchronized (synchBuffer) and released ${ }^{13}$ Once a resource is created, it can be used as input argument to setArg, to be accessed by the GPU during kernel execution.

d) Kernel invocation: Before any kernel invocations, all the arguments must be set. In addition to that, symbols and launch configuration must also be specified (resp. copySymbol $<T>$ and setLaunchConfiguration) Actual kernel invocation occurs through the launchKernel call. Internally, our implementation of $\boldsymbol{G} \boldsymbol{U S T}$ uses tables to keep track of all the previously compiled kernels, related launch configuration and layout. We recall that command list-based APIs must defer actual copy and kernel invocation as described in Sections III-C2 and IV

e) Bookkeeping: As we highlighted in the previous paragraphs, allocations, PSOs and compiled kernels are stored within internal data structures related to a compute context. Such structures are implemented as std::maps and software cache for frequently utilized resources/kernels. More specifically, the context cache remembers recently launched kernels and respective arguments to avoid table look-ups.

f) Error checking: GUST API calls are internally checked for errors. This might occur by delegating error checking to the target GPGPU API or by exploiting the bookkeeping structures. For instance, context creation failure is handled via API-specific error checking. Conversely, since compiled kernels are stored in a std: :map, attempting to call a kernel that was not previously compiled will be easily detected by simply looking up that map in our implementation.

\footnotetext{
${ }^{13}$ Due to space constraints these operations are not discussed
} 
TABLE VI: SLOC for basic compute operations. GUST compared to seven GPU APIs

\begin{tabular}{|c|c|c|c|c|c|c|c|c|}
\hline $\begin{array}{l}\text { Host Operation/ } \\
\text { API SLOC }\end{array}$ & $\begin{array}{l}\text { CUDA-Runtime } \\
\text { API }\end{array}$ & $\begin{array}{l}\text { CUDA } \\
\text { Driver API }\end{array}$ & OpenCL & OpenGL & D3D11 & D3D12 & Vulkan & GUST \\
\hline Context Creation & (implicit) & 5 & 60 & 47 & 5 & 60 & 242 & 1 \\
\hline Memory Allocations & 2 & 2 & 3 & 4 & 10 & 60 & 30 & 1 \\
\hline Memory Transfer & 1 & 1 & 1 & 4 & 10 & $\begin{array}{l}\text { (in cmd buf recording) } \\
40\end{array}$ & $\begin{array}{l}\text { (in cmd buf recording) } \\
27\end{array}$ & 1 \\
\hline Set Kernel Argument & (in kernel call) & (in kernel call) & 1 & 2 & 2 & $\begin{array}{l}\text { (in pipeline creation) } \\
20\end{array}$ & $\begin{array}{l}\text { (in pipeline creation) } \\
10\end{array}$ & 1 \\
\hline Copy Symbol & (in kernel call) & (in kernel call) & 1 & 2 & 3 & $\begin{array}{l}\text { (in cmd buf recording) } \\
2\end{array}$ & $\begin{array}{l}\text { (in cmd buf recording) } \\
27\end{array}$ & 1 \\
\hline $\begin{array}{l}\text { Set Launch } \\
\text { configuration }\end{array}$ & (in kernel call) & (in kernel call) & (in kernel call) & $\begin{array}{l}\text { (in compute } \\
\text { dispatch) }\end{array}$ & $\begin{array}{l}\text { (in compute } \\
\text { dispatch) }\end{array}$ & $\begin{array}{l}\text { (in pipeline creation) } \\
2\end{array}$ & $\begin{array}{l}\text { (in pipeline creation) } \\
20\end{array}$ & 1 \\
\hline Launch Kernel & 1 & 1 & 1 & 1 & 1 & (in cmd buf recording) & $\begin{array}{l}\text { (in cmd buf recording) } \\
2\end{array}$ & 1 \\
\hline Wait for completion & 1 & 1 & 1 & 2 & 7 & 10 & 2 & 1 \\
\hline $\begin{array}{l}\text { Kernel } \\
\text { compilation }\end{array}$ & uses nvcc & 90 (with nvrtc) & 43 & 90 & 50 & 44 & $\begin{array}{l}\text { (uses } \\
\text { glslangValidator) } 75\end{array}$ & 1 \\
\hline Submit Work & - & - & - & - & - & 1 & 1 & $\mathbf{1}$ \\
\hline Pipeline creation & - & - & - & - & - & 90 & 146 & 2 \\
\hline Cmd Buf recording & - & - & - & - & - & 4 & 4 & 2 \\
\hline
\end{tabular}

2) Device-side Operations: In our reference implementation, we opted to manage device-side translation rules and preamble definition by relying on a tag-based language that is processed by means of a source-to-source compiler as part of the compute program compilation process described in the previous section ${ }^{14}$. From the translation rules defined in tables III and $\mathrm{V}$, our reference implementation of $\boldsymbol{G} \boldsymbol{U S T}$ provides a Meta Language in which the programmer specifies arguments, symbols and local scratchpad memory allocations in a tagbased language. Then, a standard $\mathrm{C}$ program enriched with specific keywords is used to describe the parallel algorithms executed by the kernel. A source file constructed with such rules is named a MLTL (Meta Language Translation Layer) source file. The user can choose two ways of writing a compute kernel: (1) using API-specific device code; or (2) exploiting the above mentioned MLTL language. Choosing the latter requires an additional step for the compilation process, where the MLTL code is parsed and translated into the functional equivalent for the target API. Listing 2 shows a MLTL implementation of a histogram calculated over a 2D matrix of integer values. The tag based structure is evident. We can identify two tags: a first tag detailing the layout definition (LAYOUT_DEF) and a tag in which the behavior of the kernel is expressed as a standard C program (PROGRAM). Tags are delimited by the keywords between square brackets. The LAYOUT_DEF tag is composed of a forward declaration of arguments (one argument per line, starts with $A R G$ : with the syntax <name>, <usage $>\in R, W, R W\},<$ type $>$ ), symbols (one per line, starts with $S Y M$ : with the syntax <name>, <type>) and static local memory allocation (called shared, starts with SHM: with the syntax $\langle$ name $>$, <type $>,\langle e$ lements $>$ ). Layout definition is part of what is known in GUST as preamble definition. The PROGRAM tag contains standard C code defined by an entry point (a kernel identifier able to be referred from host code, in the example in Listing 2 "histo" is used). Keywords delimited by underscores refer to MLTLspecific syntax: _GTSX_ stands for block size over the X dimension, LITY $_{-}$refers to the local thread index ID over

\footnotetext{
${ }^{14}$ For an integrated compilation process, custom keywords could also be implemented in the form of language extensions (e.g., C, C++) or compiler annotations (OpenMP-style \#pragmas).
}

the $\mathrm{Y}$ dimension and so on. Fetching data from the inputs defined in the (LAYOUT_DEF) tag is done with the syntax $A R G \mid S H M$ (name, index); for symbols, $S Y M$ (name) is used. $S H M$ refers to local memory. Atomic operations and local memory barriers can be instantiated with the _ATADD_ and_LOCMEMBAR_tags, respectively. For space constraints we do not list all the possible combinations of thread indexing and synchronization currently implemented for the MLTL. The MLTL layer in $\boldsymbol{G U S T}$ is able to substitute the MLTL specific keywords in order to recreate the kernel in every APIspecific language: in doing so, our reference implementation can issue a warning or error if a violation of the MLTL-specific syntax was detected during source translation. Non-MLTLrelated errors will be flagged by the chosen API library during native compilation.

\section{An Early Assessment of $\boldsymbol{G U S T}$}

We assess the benefits of the proposed unified specification in terms of ease of development and performance penalty (overhead characterization). Performance hit is also qualitatively discussed for the unification of traditional and command list-based APIs.

1) Ease of development: The abstraction provided by GUST translates in a simplified coding style, which we quantify by means of source lines of code (SLOC). SLOC for each API compared to $\boldsymbol{G U S T}$ is visible in Table VI Here, we highlighted how many lines of code are needed for each operation abstracted by our reference $\boldsymbol{G} \boldsymbol{U S T}$ implementation: for each API, the line count includes basic error checking and bookkeeping operations. D3D12 and Vulkan are intuitively expected to require a similar line code count, but this is not observed in table VI] This is due to the fact that most of D3D12 structure initialization occurs through a helper library function (D3DX12) which moderates coding verbosity in Direct Compute applications.

From Table VI is evident that $\boldsymbol{G} \boldsymbol{U S T}$ is very effective at reducing the amount of code to be written for GPU application development (in the compute pipeline), as most of the functionalities are wrapped within a single function call. We also include numbers from a seventh API, the CUDA 
runtime $A P I$, which is not wrapped by our implementation of $\boldsymbol{G U S T}$, but provides an interesting term of comparison since it is in practice the most used GPGPU API, due to its ease of use. Compared to command list-based APIs the reduction in SLOC for $\boldsymbol{G U S T}$ is major. On the device side, for MLTL there is basically a 1:1 translation for every construct to any back-end API, which implies no increase in SLOC. Only what we call the MLTL preamble slightly increases the instruction count compared to CUDA and OpenCL. This is because while CUDA and OpenCL describe kernel inputs and output buffers in the device function signature, in GLSL and HLSL (used for the other APIs) inputs and outputs are specified in a separate code block: for simplicity our MLTL prototype translator matches the GLSL/HLSL specification.

In $\boldsymbol{G} \boldsymbol{U S T}$, porting one application from an API to another is simple, provided that both the starting API and the destination API belong to the same category (traditional and command list-based). The only part that would require a light programming effort is instantiating the compute context: some API-specific settings might be necessary to be specified as context constructor parameters. However, default constructors passing default settings can be easily put in place. Moreover, it is trivial to automate the selection of the most appropriate API backend in $\boldsymbol{G} \boldsymbol{U S T}$ during context creation as information such as device vendor and installed drivers can be easily queried at runtime. Device code written in MLTL does not require any modification. The possibility to port the host code of an API belonging to a category to a target API belonging to a different category is discussed in section IV-C3

2) Overhead characterization: The overhead characterization w.r.t. the APIs wrapped by $\boldsymbol{G} \boldsymbol{U S T}$ depends of the design choices of the $\boldsymbol{G} \boldsymbol{U} \boldsymbol{S} \boldsymbol{T}$ implementer; our reference implementation heavily relies on red-black tree-based maps to perform state tracking and bookkeeping of host-side constructs like compiled shaders and allocation tables. Every time the $\boldsymbol{G} \boldsymbol{U S T}$ user attempts to use a kernel with specific resources, look-up operations on all these maps are triggered. Overhead given by the look-up operations is therefore related to how many kernels and allocations are distinctively used within the same application. Since we used regular std::maps, look-up complexity is logarithmic in size; moreover, recently used kernels and layouts are cached to local variables to amortize look-up costs for complex applications. On the device side, our reference implementation adds no overhead other than the compilation time from MLTL to API-specific device code.

However, it is still interesting to measure how $\boldsymbol{G} \boldsymbol{U S T}$ performs in terms of overhead compared to the only API that is not directly targeted: the CUDA Runtime API. For this experiment, $\boldsymbol{G} \boldsymbol{U} \boldsymbol{S} \boldsymbol{T}$ is set to wrap the CUDA driver API. Results on a small set of benchmarks are reported in Figure 2. The benchmarks include a Vector Addition (VADD), SinglePrecision $A \times X+Y$ (SAXPY), 2D histogram computation (HISTO) and a parallel reduction on an array of integers (MINREDUX). Dataset size is $512 \mathrm{~K}$ elements for each buffer in SAXPY, VADD and MINREDUX. Histogram is computed on a 256 -colors image $(1024 \times 768)$. Although all the benchmarks call all the GUST functions listed in Tables IV and $\mathrm{V}$ we also provide an experiment with MINREDUX where we study the effect of varying the input dataset size, and we provide a fifth benchmark, a 2D Finite Difference Time Domain solver ${ }^{15}$ (FDTD2D). FDTD2D represents a more complex application composed of different kernels that are iterated multiple (500) times. The dataset consists of $2048 \times 2048$ floating point values. All The tests are executed on a Intel i7 x86_64 platform featuring a GTX860M NVIDIA discrete GPU.

The leftmost plot in Figure 2 shows execution times for GUST and the CUDA runtime API (normalized to the latter). Execution times include allocations, data initializations, memory transfers (any direction), kernel launches and hostdevice synchronization operations. In all the tested benchmarks $\boldsymbol{G} \boldsymbol{U} \boldsymbol{S} \boldsymbol{T}$ performs on par with the CUDA runtime API (within $\pm 0.04 \%$ ). This is not unexpected, as both $\boldsymbol{G U S T}$ and the CUDA runtime eventually make the same calls to the underlying CUDA Driver API functions, as it's easily confirmed running the CUDA profiler, nvprof.

Overhead impact is also independent of the dataset size, as shown by our experiment with MINREDUX in the center plot in Figure 2). Varying the dataset size from $512 \mathrm{~K}$ to $10 \mathrm{M}$ integer elements does not significantly affect execution time. This is also intuitively explained by the fact that bookkeeping operations are only sensitive to the number of buffers used in a program, not their size. The larger the dataset, the longer the kernel processing time and thus the less relevant the already negligible host-side overheads.

The rightmost plot in Figure 2 shows the overhead implied by the MLTL compilation step occurring before native, API-specific back-end compilation. Specifically, this overhead is calculated as $\frac{T_{G U S T \text { compile }}+T_{N V R T C \text { compile }}}{T_{N V R T C \text { compile }}}$ where $T_{\text {GUSTcompile }}$ is the time to translate MLTL to CUDA and $T_{N V R T C c o m p i l e}$ is the native CUDA compilation time using NVRTC. Again, this overhead is largely implementationspecific, but even with our proof-of-concept translator it is easy to see that this overhead is negligible, as MLTL only supports a small subset of device-side constructs and only operates simple line-by-line translation, leaving more sophisticated optimizations via full-blown compiler intermediate representation (IR) to the following API-specific compilation steps.

3) Unifying API categories: In the methodology we have presented we kept a distinction between traditional APIs and command list-based APIs, due to the different application development philosophy underlying the two. However, nonexpert developers dealing with an application port that targets a command list-based API might still find it very useful to stick to a simple, unified specification layer. To support this, it is possible to define mechanisms for just-in-time creation of PSOs and command lists starting from a program that exploits the traditional API coding style. We discuss two strategies: (i) lazy PSO and command buffer creation and (ii) command batching from static analysis.

${ }^{15}$ From the Polybench benchmark suite: http://web.cs.ucla.edu/ pouchet/ software/polybench/ 

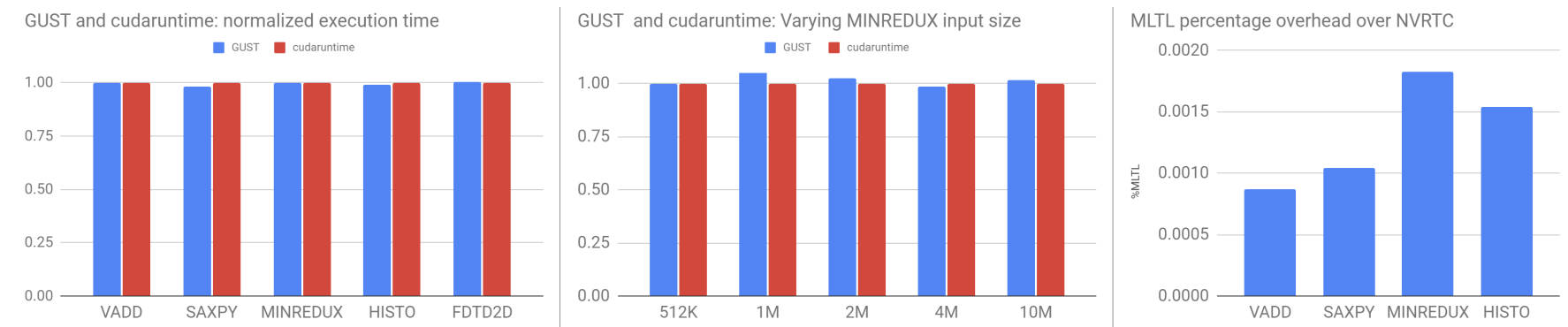

Fig. 2: GUST prototype implementation vs. CUDA Runtime API. Average execution time (left). Effect of varying dataset size in the MINREDUX benchmark (center). MLTL compilation overhead over total compilation time (right).

a) Lazy PSO and command buffer creation: - Starting from a $\boldsymbol{G U S T}$ application written using the traditional API coding style, each kernel call is intercepted at runtime: a corresponding PSO is created on the fly according to the kernel invoked, its layout and its launch configuration. Such PSO is hashed and stored in a cache, so to avoid recreating the same PSO in case of reuse. vudd ${ }^{16}$ a coding effort that enables the user to write CUDA code over a Vulkan implementation, follows this blueprint. Memory transfers and actual kernel launches will have to be recorded in a command buffer: this introduces the problem of when to start the recording phase and when to stop it, to then submit the command buffer to the GPU. This is because the workload might be composed of a sequence of an unknown number of copy and compute commands. This can be solved by recording a command buffer for each operation within the sequence, and offloading the work as soon as the recording phase terminates. Another strategy for managing the command buffer is to start the recording upon the first kernel launch or copy invocation and terminate the recording phase when the application calls a GPU-CPU synchronization point (e.g: wait for idle) or when a read-back of a resource used by the GPU is requested by the host. While the first strategy is very easy to implement, the resulting performance hit can be dramatic for long GPU command sequences, especially if the GPU commands submitted present small execution times. The second strategy allows $\boldsymbol{G} \boldsymbol{U} \boldsymbol{S T}$ to batch many commands within a single command buffer recording phase so to minimize submission operations. This is optimal, but implementationwise a variety of problems arise: in GPGPU computing calls to CPU-GPU synchronization points are not mandatory, and the read back resources by the host can occur via multiple device-to-host copy commands. These are the uncertainties to face when deciding to close the recording phase of a command buffer. Heuristics based on time windows might be used to mitigate these issues: the first copy or dispatch command signals the beginning of a recording phase and all the commands invoked within a pre-determined time window will end up in the same command submission. A third possible strategy exploits CUDA graphs: a feature introduced in CUDA 8. CUDA graphs enqueue GPU commands with pre-defined precedence constraints. Building a CUDA graph is semantically very similar to recording a command buffer, as intended

\footnotetext{
${ }^{16}$ https://github.com/jgbit/vuda
}

in next-generation APIs. Although this feature is designed for reducing submission overheads, offloading commands in such a way might still result in higher overheads than submitting a command buffer using next-generation APIs [31].

b) Command batching from static analysis: - Static code analysis can theoretically improve upon the cost for just-intime creation of PSOs and help in the selection of the right heuristics for command buffer recording. Creating a PSO for each kernel (including layout definition and launch configuration) takes $20 \mathrm{~ms}$ in an NVIDIA Jetson AGX embedded board using Vulkan. In the same board, recording a command buffer takes a constant time of $2.5 \mathrm{~ms}$ for sequences of less then 100 copy and compute commands; after that, the time needed for recording a command list scales linearly [31]. Trivially, for the examined board, recording and submitting a command list for each copy or compute command is convenient for GPU execution times larger than the time needed to finalize the command list for each command. In this way, CPU execution times for the recording phase can proceed in parallel with GPU execution. If using an approach based on time windows it is instead convenient that the length of the time window is sized as a negligible value compared to the sum of the execution times of the commands batched within the time window. Command lists can be hashed and cached just like PSOs for optimizing submission operations in case of periodic workloads.

\section{Final Discussion and Conclusion}

In this paper we presented an exhaustive overview on the most commonly used GPU APIs for general purpose computing. From there we derived a taxonomy with two macro categories: traditional APIs (CUDA, OpenCL, OpenGL compute shaders, DirectCompute over Direct3D11) and command list-based APIs (Vulkan and DirectCompute over Direct3D12 APIs). Among APIs belonging in the same category we identified the subset of programming constructs that describe the same functionality, both for host- and device-side code. This allowed us to define a unified specification across the various APIs, which is sufficiently expressive to capture the basic blocks of any heterogeneous programming application targeting a GPGPU as an accelerator. We provide a reference implementation of such specification, called $\boldsymbol{G} \boldsymbol{U S T}$, which we use to validate the benefits of the approach. Our unified specification and the $\boldsymbol{G} \boldsymbol{U} \boldsymbol{S T}$ tool proved to be very effective at porting 
applications onto different platforms, simplifying both application development and extensive cross-benchmarking [31]. Performance-wise, we have observed minimal overheads for the abstraction layer.

$\boldsymbol{G U S T}$ is meant as a cross-platform API specification, yet some of the APIs remain hardware- or operating systemspecific (e.g., D3D wrappers only work on Windows; CUDA is specific to NVIDIA hardware). Trivially, there is no workaround for this: the only APIs known to be completely cross-platform are the ones defined by the Khronos specifications. However, the proposed methodology supports all such standards, and identifies at least two alternatives for all the common operating system and hardware configurations found in the market, for both desktop and embedded systems.

As of now, $\boldsymbol{G} \boldsymbol{U} \boldsymbol{S T}$ is far from being feature-complete. In particular, some relevant differences between the APIs complicate the unification of the specification. Today, our reference implementation manages this functionality gap by allowing the experienced developer to write algorithms in MLTL syntax, so to have a translation to all the other APIs specific device-code. Then, through manual tuning, the developer is still able to use API-specific features for optimization.

As future work, we plan to incrementally add to our reference implementation additional features, such as support for unified memory models, parallel submission of command buffers (in the form of multiple queue of commands), multiGPU support and the implementation of a unifying layer between traditional and command list-based APIs (as discussed in section IV-C3. Our reference implementation of $\boldsymbol{G} \boldsymbol{U S T}$ will soon be released as an open source contribution. The interested reader can find $\boldsymbol{G} \boldsymbol{U} \boldsymbol{S} \boldsymbol{T}$ interface sources together with a Vulkan implementation at this link https://git.hipert.unimore. it/rcavicchioli/cpu_gpu_submission/-/tree/master/vkcomp

\section{ACKNOWLEDGEMENT}

This work has received funding from EU projects $\mathrm{H} 2020$ CLASS (780622) and ECSEL JU NEWCONTROL (826653) and COMP4DRONES (826610).

\section{REFERENCES}

[1] A. Sridhar, A. Vincenzi, M. Ruggiero, and D. Atienza, "Neural Network-Based Thermal Simulation of Integrated Circuits on GPUs," IEEE Transactions on Computer-Aided Design of Integrated Circuits and Systems, vol. 31, no. 1, pp. 23-36, 2012.

[2] E. Schneider and H. Wunderlich, "SWIFT: SwitchLevel Fault Simulation on GPUs," IEEE Transactions on Computer-Aided Design of Integrated Circuits and Systems, vol. 38, no. 1, pp. 122-135, 2019.

[3] Y. Lin, Z. Jiang, J. Gu, W. Li, S. Dhar, H. Ren, B. Khailany, and D. Z. Pan, "DREAMPlace: Deep Learning Toolkit-Enabled GPU Acceleration for Modern VLSI Placement," IEEE Transactions on Computer-Aided Design of Integrated Circuits and Systems, pp. 1-1, 2020.

[4] B. Shi, Y. Zhang, and A. Srivastava, "Accelerating Gate Sizing Using Graphics Processing Units," IEEE Transactions on Computer-Aided Design of Integrated Circuits and Systems, vol. 31, no. 1, pp. 160-164, 2012.
[5] M. Ujaldon and U. V. Catalyurek, "High-performance signal processing on emerging many-core architectures using CUDA," in 2009 IEEE International Conference on Multimedia and Expo. IEEE, 2009, pp. 1825-1828.

[6] S. R. Upadhyaya, "Parallel approaches to machine learning-A comprehensive survey," Journal of Parallel and Distributed Computing, vol. 73, no. 3, pp. 284-292, 2013.

[7] S. Mittal and J. S. Vetter, "A survey of CPU-GPU heterogeneous computing techniques," ACM Computing Surveys (CSUR), vol. 47, no. 4, p. 69, 2015.

[8] N. Rajovic, A. Rico, J. Vipond, I. Gelado, N. Puzovic, and A. Ramirez, "Experiences with mobile processors for energy efficient HPC," in 2013 Design, Automation \& Test in Europe Conference \& Exhibition (DATE). IEEE, 2013, pp. 464-468.

[9] P. Faber and A. Größlinger, "A comparison of GPGPU computing frameworks on embedded systems," IFACPapersOnLine, vol. 48, no. 4, pp. 240-245, 2015.

[10] S. Schaetz and M. Uecker, "A multi-GPU programming library for real-time applications," in International Conference on Algorithms and Architectures for Parallel Processing. Springer, 2012, pp. 114-128.

[11] J. Enmyren and C. W. Kessler, "Skepu: a multi-backend skeleton programming library for multi-gpu systems," in Proceedings of the fourth international workshop on High-level parallel programming and applications, 2010, pp. 5-14.

[12] D. A. Beckingsale, J. Burmark, R. Hornung, H. Jones, W. Killian, A. J. Kunen, O. Pearce, P. Robinson, B. S. Ryujin, and T. R. Scogland, "Raja: Portable performance for large-scale scientific applications," in 2019 ieee/acm international workshop on performance, portability and productivity in hpc (p3hpc). IEEE, 2019, pp. 71-81.

[13] T. Heller, P. Diehl, Z. Byerly, J. Biddiscombe, and H. Kaiser, "Hpx-an open source c++ standard library for parallelism and concurrency," Proceedings of OpenSuCo, vol. 5, 2017.

[14] H. C. Edwards and C. R. Trott, "Kokkos: Enabling performance portability across manycore architectures," in 2013 Extreme Scaling Workshop (xsw 2013). IEEE, 2013, pp. 18-24.

[15] S. Memeti, L. Li, S. Pllana, J. Kołodziej, and C. Kessler, "Benchmarking OpenCL, OpenACC, OpenMP, and CUDA: programming productivity, performance, and energy consumption," in Proceedings of the 2017 Workshop on Adaptive Resource Management and Scheduling for Cloud Computing. ACM, 2017, pp. 1-6.

[16] A. Acosta, C. Merino, and J. Totz, "Analysis of OpenCL Support for Mobile GPUs on Android," in Proceedings of the International Workshop on OpenCL. ACM, 2018, p. 27.

[17] S. Kim and S.-K. Kim, "Comparison of OpenCL and RenderScript for mobile devices," Multimedia Tools and Applications, vol. 75, no. 22, pp. 14 161-14 179, 2016.

[18] R. Membarth, O. Reiche, F. Hannig, and J. Teich, "Code generation for embedded heterogeneous architectures on Android," in 2014 Design, Automation \& Test in Europe 
Conference \& Exhibition (DATE). IEEE, 2014, pp. 1-6.

[19] M. Bourgoin, E. Chailloux, and J.-L. Lamotte, "Efficient abstractions for GPGPU programming," International Journal of Parallel Programming, vol. 42, no. 4, pp. 583600, 2014.

[20] A. Mazaheri, J. Schulte, M. W. Moskewicz, F. Wolf, and A. Jannesari, "Enhancing the Programmability and Performance Portability of GPU Tensor Operations," in European Conference on Parallel Processing. Springer, 2019, pp. 213-226.

[21] Y. Yang, P. Xiang, J. Kong, M. Mantor, and H. Zhou, "A unified optimizing compiler framework for different GPGPU architectures," ACM Transactions on Architecture and Code Optimization (TACO), vol. 9, no. 2, p. 9, 2012.

[22] E. Holk, M. Pathirage, A. Chauhan, A. Lumsdaine, and N. D. Matsakis, "GPU programming in rust: Implementing high-level abstractions in a systems-level language," in 2013 IEEE International Symposium on Parallel \& Distributed Processing, Workshops and Phd Forum. IEEE, 2013, pp. 315-324.

[23] X. Tian, R. Xu, Y. Yan, Z. Yun, S. Chandrasekaran, and B. Chapman, "Compiling a high-level directivebased programming model for gpgpus," in International Workshop on Languages and Compilers for Parallel Computing. Springer, 2013, pp. 105-120.

[24] R. Reyes, I. López-Rodríguez, J. J. Fumero, and F. De Sande, "accULL: an OpenACC implementation with CUDA and OpenCL support," in European Conference on Parallel Processing. Springer, 2012, pp. 871882.

[25] D. Sharlet, A. Kunze, S. Junkins, and D. Joshi, "Shevlin park: Implementing c++ amp with clang/llvm and opencl," in General Meeting of LLVM developers and users, 2012.

[26] J. Fang, A. L. Varbanescu, and H. Sips, "A comprehensive performance comparison of CUDA and OpenCL," in 2011 International Conference on Parallel Processing. IEEE, 2011, pp. 216-225.

[27] P. Du, R. Weber, P. Luszczek, S. Tomov, G. Peterson, and J. Dongarra, "From CUDA to OpenCL: Towards a performance-portable solution for multi-platform GPU programming," Parallel Computing, vol. 38, no. 8, pp. 391-407, 2012.

[28] A. Rao, A. Srivastava, K. Yogesh, A. Douillet, G. Gerfin, M. Kaushik, N. Shulga, V. Venkataraman, D. Fontaine, M. Hairgrove et al., "Unified memory systems and methods," Jul. 23 2015, uS Patent App. 14/601,223.

[29] M. Knap and P. Czarnul, "Performance evaluation of Unified Memory with prefetching and oversubscription for selected parallel CUDA applications on NVIDIA Pascal and Volta GPUs," The Journal of Supercomputing, pp. 1-21, 2019.

[30] J. Nickolls, I. Buck, and M. Garland, "Scalable parallel programming," in 2008 IEEE Hot Chips 20 Symposium (HCS). IEEE, 2008, pp. 40-53.

[31] R. Cavicchioli, N. Capodieci, M. Solieri, and M. Bertogna, "Novel Methodologies for Predictable
CPU-To-GPU Command Offloading," in 31st Euromicro Conference on Real-Time Systems (ECRTS 2019). Schloss Dagstuhl-Leibniz-Zentrum fuer Informatik, 2019.

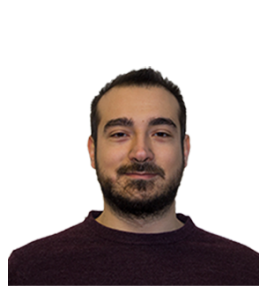

Nicola Capodieci is an associate researcher at the HiPeRT-Lab of the University of Modena and Reggio Emilia. His main research interests range from distributed systems to languages, architectures and programming models for GPUs.

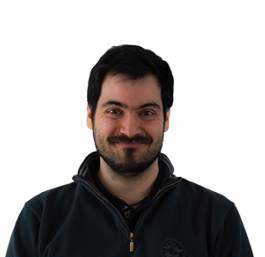

Roberto Cavicchioli Roberto Cavicchioli is a PostDoctoral Researcher at the HiPeRT-Lab (University of Modena and Reggio Emilia) whose research is focused on parallel computing for heterogeneous systems, optimization algorithms, machine learning and real time scheduling. He received his Ph.D. in 2014.

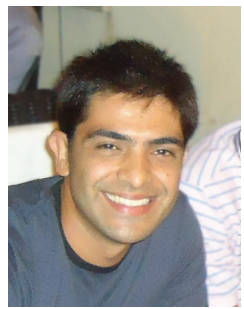

Andrea Marongiu received the $\mathrm{PhD}$ degree in electronic engineering from the University of Bologna, Italy, in 2010. He has been a postdoctoral reserch fellow at ETH Zurich, Switzerland. He currently is an associate professor at the University of Modena and Reggio Emilia. His research interests focus on programming models and architectures in the domain of heterogeneous multi- and many-core systems-onchip. In this field, he has published more than 100 papers in peer-reviewed conferences and journals. 